\title{
Prognostic Factors in Patients with Distant Soft Tissue Metastasis of Carcinoma: A Clinicopathological Study of 16 Cases
}

\author{
Hiroyuki Tsuchie $^{a} \quad$ Makoto Emori $^{\mathrm{b}}$ Naohisa Miyakoshi ${ }^{\mathrm{a}}$ Kyoji Okadac

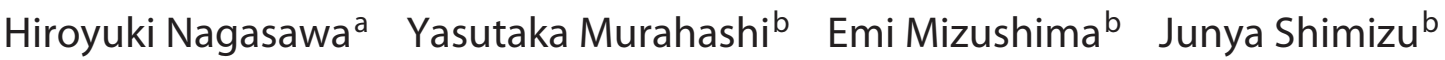 \\ Toshihiko Yamashitab Yoichi Shimada ${ }^{\mathrm{a}}$ \\ a Department of Orthopedic Surgery, Akita University Graduate School of Medicine, Akita, Japan; \\ ${ }^{b}$ Department of Orthopedic Surgery, Sapporo Medical University School of Medicine, Sapporo, Japan; \\ 'Department of Physical Therapy, Akita University Graduate School of Health Sciences, Akita, Japan
}

\section{Highlights of the Study}

- Soft tissue metastasis (STM) is an uncommon condition in carcinoma, and its prognostic factors remain unclear.

- We found that no past treatment of the primary tumor and absence of pain were poor prognostic factors in STM.

- STM in the deep layer is at high risk of being painful.

\section{Keywords}

Metastasis · Soft tissue $\cdot$ Carcinoma

\begin{abstract}
Objectives: Soft tissue metastasis (STM) is an uncommon condition in carcinoma. Although various case series related to STM have been reported, few reports have examined prognostic factors. This study aimed to evaluate the characteristics of STM and the factors affecting its prognosis. Materials and Methods: Patients with STM from carcinoma were retrospectively studied. The patients' information, including age, sex, primary tumor, metastasis location, size of the metastatic tumor, presence of pain, histological classification, history of primary tumor treatment, and other metastasis at diagnosis of STM were collected and associated with prognosis. Results: Overall, 16 patients with a mean age of 68.7
\end{abstract}

karger@karger.com www.karger.com/mpp

Karger $\frac{1}{\%}$

GOPEN ACCESS
(C) 2020 The Author(s)

Published by S. Karger AG, Basel

This is an Open Access article licensed under the Creative Commons Attribution-NonCommercial-4.0 International License (CC BY-NC) (http://www.karger.com/Services/OpenAccessLicense), applicable to the online version of the article only. Usage and distribution for commercial purposes requires written permission. years were evaluated. The overall survival rate was not significantly different between lung cancer and non-lung cancer patients. The overall survival rate was significantly better in patients undergoing definitive treatment for the primary tumor than in those without history of treatment $(p=0.046)$. The overall survival rate of STM patients with no metastasis was significantly better than those with other metastasis at the diagnosis of STM $(p=0.041)$. On multivariate analysis, no history of primary tumor treatment and STM without pain were risk factors for prognosis $(p=0.0340$ and 0.0474 , respectively). None of the patients who developed STM under the skin experienced pain, while $92.3 \%$ of the patients who developed STM in the deep layer had pain. Conclusion: The risk factors for poor diagnosis of STM were no past treatment of the primary tumor and absence of pain. STM in the deep layer is prone to pain.

(C) 2020 The Author(s) Published by S. Karger AG, Basel 


\section{Introduction}

The incidence of cancer has been gradually increasing in recent years. In 2018, the World Health Organization (WHO) reported 18.1 million new cancer cases worldwide; approximately 1 million were from Japan $[1,2]$. Developments in cancer treatment including new chemotherapeutics, new surgical techniques, and heavy-particle radiotherapy have prolonged patient survival; however, this has also led to an increase in the number of patients with metastasis. Therefore, the diagnosis and treatment for metastasis have gained particular importance.

Metastases from carcinomas usually present at regional lymph nodes and other organs including the liver and lung; the soft tissue is an uncommon site for metastasis owing to specific homeostatic conditions that suppress tumor cell proliferation, constant trauma to cells due to muscle contraction, lactic acid production by the skeletal muscle, and high tumor cell permeability [3]. Although various case reports and series have been reported with regard to soft tissue metastasis (STM) of cancer, large-scale studies are lacking [4-18]. In addition, most of the previous reports have only considered their clinical trends and diagnostic methods to distinguish them from soft tissue sarcoma, and few studies have examined prognostic factors. Therefore, this study aimed to analyze the characteristics of STM and the factors affecting its prognosis.

\section{Subjects and Methods}

\section{Subjects}

This retrospective study enrolled patients with STM from carcinoma who were diagnosed and treated in 2 hospitals between 1994 and 2018. We reviewed their medical records retrospectively, and 16 consecutive patients who had sufficient medical records were included. Those with distant metastases that developed in the subcutaneous tissue or skeletal muscle were included, while those with needle tract metastases after biopsy, metastases to reactive area around wounds exposed at the time of primary tumor excision, and direct extension from adjacent tumors were excluded. The patients' information, including age, sex, primary tumor, metastasis location, size of the metastatic tumor, presence of pain, histological classification, history of primary tumor treatment, other metastasis at diagnosis of STM, follow-up period, and outcomes were collected. We defined definitive treatment for primary tumor as complete surgical resection.

\section{Prognostic Factors}

In this study, we examined the factors that may affect prognosis and compared their predictive value for prognosis. Primary tumor site and histological type, history of primary tumor treatment, and other metastasis at diagnosis were included in the analysis.

Prognostic Factors in Patients with

Distant Soft Tissue Metastasis
Table 1. Clinical data of 16 patients with soft tissue metastasis of carcinoma

$N$

Age, years 16

Male/female $68.7 \pm 13.2$

Primary tumor, lung/not lung

$12 / 4$

Metastasis location, extremity/axi

$6 / 10$

$11 / 5$

Depth, subcutaneous/muscle

$3 / 13$

Size, $\mathrm{mm}$

$84.8 \pm 29.5$

Pain, present/none

$12 / 4$

Histological type

Adenocarcinoma 9

Squamous cell carcinoma

Undifferentiated carcinoma

5

2

$6 / 10$

Past primary tumor treatment, present/none 6/10

Other metastasis at diagnosis of STM, present/none $\quad 10 / 6$

Surgical treatment for soft tissue metastasis, present/none

Radiation, present/none $\quad 8 / 8$

Follow-up period, months $11.8 \pm 12$.

Outcome at the last follow-up

Alive with disease 3

Dead 13

Values are expressed as number of patients or mean \pm SD. STM, soft tissue metastasis.

\section{Statistical Analysis}

Overall survival (OS) was defined as the time period from the date of diagnosis to the date of death or the last follow-up. In the absence of any events, the date of the last follow-up was considered as the endpoint. As there were no deaths due to postoperative complications in this study, we defined the death from original disease as "died of disease".

All continuous variables were expressed as means \pm SDs. OS curves were drawn using the Kaplan-Meier method, and differences were analyzed using the generalized Wilcoxon test. A Cox proportional hazards model was used to identify the factors associated with prognosis. Results with $p$ values $<0.05$ were considered significant.

\section{Results}

In total, 16 patients ( 12 males and 4 females) with a mean age of 68.9 years (range $45-89$ ) were included in the study. The clinicodemographic characteristics of the patients are shown in Table 1 . The most common site of the primary tumor was the lung ( $n=6$ patients), followed by the uterus ( $n=2$ patients). The primary tumor was located in the esophagus, stomach, colon, rectum, pancreas, bladder, maxilla, and had an unknown origin in 1 patient each; 4, 7, and 5 STMs were located 
Fig. 1. Kaplan-Meier OS curves according to regions of the primary tumor. There is no significant difference between lung and non-lung cancers $(p=0.957)$.

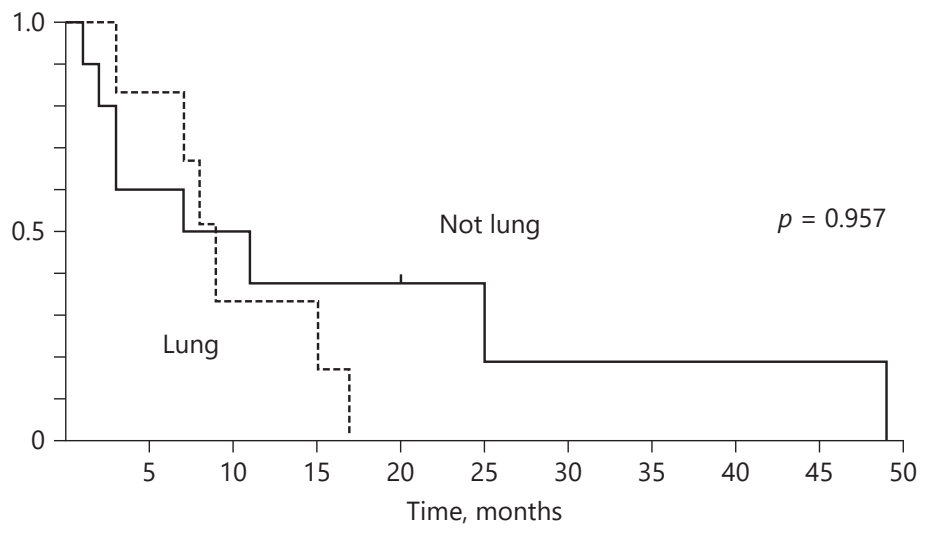

Number at risk

$\begin{array}{llllllllllll}\text { Lung } & 6 & 5 & 2 & 2 & 0 & 0 & 0 & 0 & 0 & 0 & 0\end{array}$

$\begin{array}{llllllllllll}\text { Not lung } & 10 & 6 & 4 & 3 & 3 & 2 & 1 & 1 & 1 & 1 & 0\end{array}$
Fig. 2. Kaplan-Meier OS curves according to histological type. There is no significant difference between adenocarcinoma and squamous cell carcinoma $(p=0.837)$.

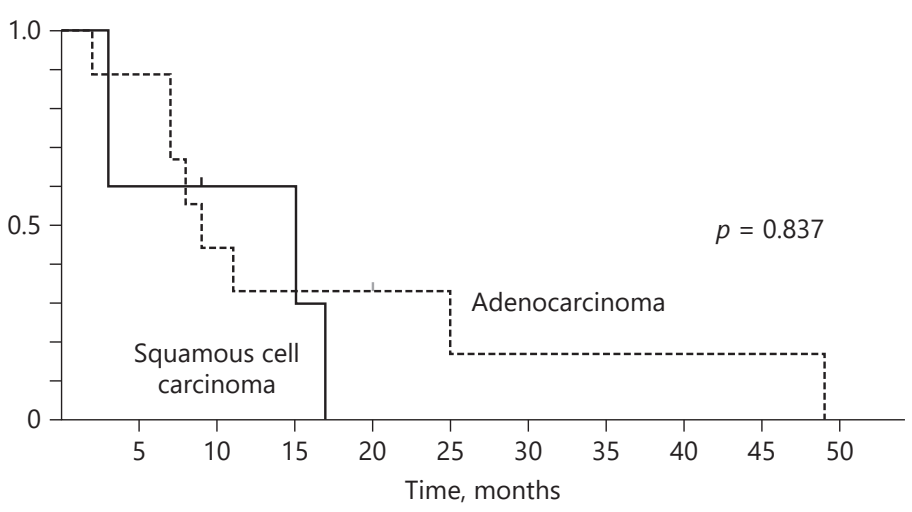

Number at risk

$\begin{array}{llllllllllll}\text { Adenocarcinoma } & 9 & 8 & 4 & 3 & 3 & 2 & 1 & 1 & 1 & 1 & 1\end{array}$

$\begin{array}{llllllllllll}\text { Squamous cell } & 5 & 3 & 2 & 2 & 0 & 0 & 0 & 0 & 0 & 0 & 0\end{array}$ in the upper extremities, lower extremities, and in the trunk, respectively. The majority of STMs developed in the deep layers $(81.3 \%)$; these tended to induce pain (75\%). None of the patients who developed STM under the skin developed pain; conversely, $92.3 \%$ of the patients who developed STM in the deep layer experienced pain. The mean radiological size of the tumors was $84.8 \mathrm{~mm}$ (range 30-121). Adenocarcinoma was the most common histological type of the primary tumor. Approximately one-third of the patients (37.5\%) received a definitive treatment for the primary tumor before developing STM, and the mean period from the treatment for the primary tumor to the onset of STM was 77 months (range 4-336). Ten patients (62.5\%) had distant metastases other than STM at diagnosis; of these, 4 were in the lung, 3 in the bone, 2 each in the adrenal gland, liver, and brain, and 1 each in the retroperitoneum, small intestine, and face. An adequate tumorfree margin was obtained in 1 patient who underwent surgical resection for STM. Five patients (31.3\%) developed new metastasis after the first diagnosis of STM; 3 were in the brain and 2 in the bone. The median followup period was 11.8 months (range 1-49).

The OS rate was not significantly different between lung and non-lung cancer patients and between adenocarcinoma and squamous cell carcinoma histologies 
Fig. 3. Kaplan-Meier OS curves according to past treatment history of the primary tumor. A significant difference is shown between those who did and did not receive treatment for the primary tumor $(p=$ 0.046).

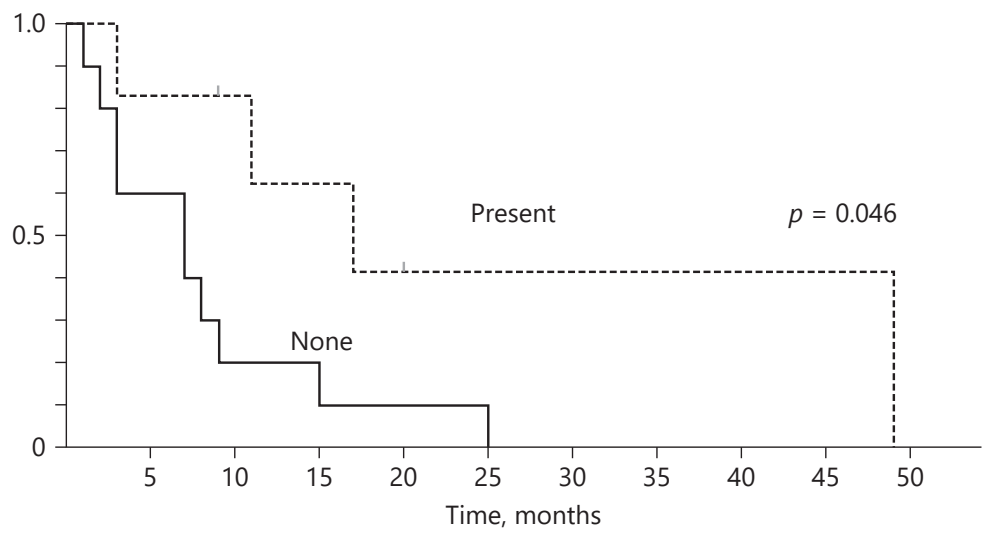

Number at risk

$\begin{array}{llllllllllll}\text { Present } & 6 & 5 & 4 & 3 & 2 & 1 & 1 & 1 & 1 & 1 & 1\end{array}$

$\begin{array}{llllllllllll}\text { None } & 10 & 6 & 2 & 2 & 1 & 1 & 0 & 0 & 0 & 0 & 0\end{array}$

Fig. 4. Kaplan-Meier OS curves according to other metastasis at STM diagnosis. A significant difference is shown between those who had and did not have other metastasis at the time of STM diagnosis $(p=0.041)$.

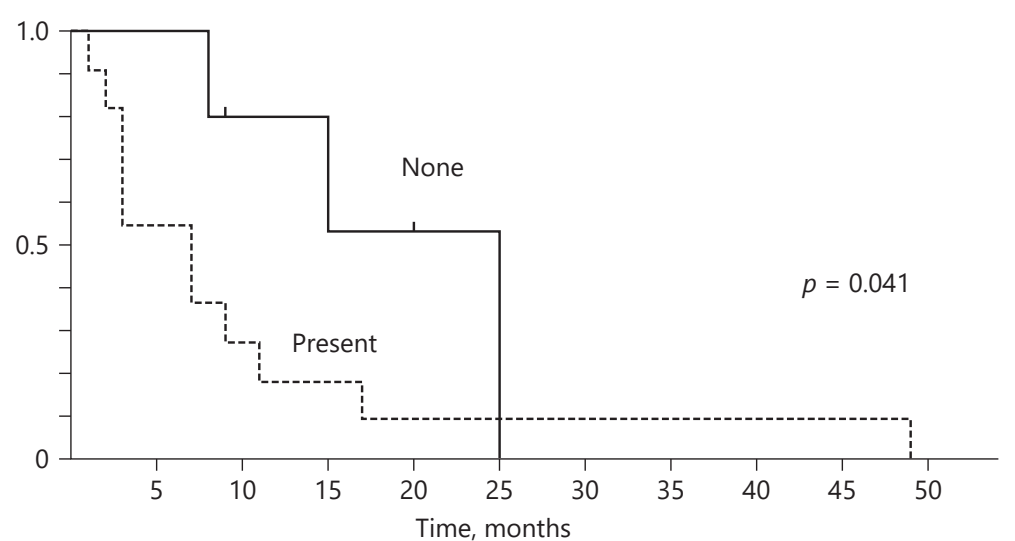

Number at risk

$\begin{array}{lllllllllll}\text { Present } 11 & 6 & 3 & 2 & 1 & 1 & 1 & 1 & 1 & 1 & 1\end{array}$

None $\begin{array}{lllllllllll}5 & 5 & 3 & 3 & 2 & 1 & 0 & 0 & 0 & 0 & 0\end{array}$
(Fig. 1, 2). The OS rate was significantly better in patients who received definitive treatment for the primary tumor than in those without history of treatment $(p=$ 0.046 , Fig. 3). The OS rate of STM patients without any metastasis was significantly better than that of those with other metastases at the diagnosis of STM $(p=0.041$, Fig. 4). On multivariate analysis for factors associated with prognosis, (i) no history of primary tumor treatment and (ii) STM without pain were identified as risk factors for prognosis ( $p=0.0340$ and 0.0474 , respectively; Table 2).

Prognostic Factors in Patients with Distant Soft Tissue Metastasis

\section{Discussion}

In our study, no past treatment of the primary tumor was found to be a poor prognostic factor for STM. Herring et al. [9] reported that there was no significant difference in prognosis between STM diagnosed before and after primary tumor diagnosis. However, they only evaluated the past diagnosis and survival times of primary tumors. Various clinical factors, such as age, location, and tumor size, may also generally affect prognosis; therefore, these factors should be included when evaluating prognosis. In addition, in the co- 
Table 2. Multivariate analysis of the factors affecting prognosis

\begin{tabular}{lrll}
\hline Variables & OR & $95 \%$ CI & $p$ value \\
\hline Age, years & 0.888 & $0.753-1.047$ & 0.1584 \\
Female & 0.017 & $0.000-21.14$ & 0.2610 \\
Location, axial & 28.217 & $0.379-2100$ & 0.1288 \\
Size & 1.090 & $0.986-1.204$ & 0.0909 \\
Primary tumor, lung & 0.027 & $0.000-1.648$ & 0.0852 \\
Past primary tumor treatment & 0.015 & $0.000-0.729$ & 0.0340 \\
Pain & 0.002 & $0.000-0.930$ & 0.0474 \\
Histological type, adenocarcinoma & 0.757 & $0.043-13.24$ & 0.8487 \\
Other metastasis at diagnosis of STM13.22 & $0.974-179.3$ & 0.0523 \\
Surgical treatment for metastasis & & & \\
$\quad$ lesions in soft tissue & 0.700 & $0.024-20.86$ & 0.8370
\end{tabular}

OR, odds ratio; 95\% CI, 95\% confidence interval; STM, soft tissue metastasis.

hort studied by Herring et al. [9], it is unclear whether the primary tumor was treated before the diagnosis of STM; all patients who were previously diagnosed with primary tumors were treated in our current study. In addition, in the study by Herring et al. [9], the time from the diagnosis of the primary lesion to the detection of STM is unknown. If STM is diagnosed after detection of the primary tumor, the prognosis is relatively good, and this may be attributed to the aggressiveness and speed of tumor invasion.

Although many case reports on STM are available, these are limited to $<10$ cases [4-18]. Previous reports have shown that primary tumors are more common in lung cancer, and histologic types are more common in adenocarcinoma; our study demonstrated the same results. Only 2 reports have examined the factors affecting prognosis in patients with STM [8, 9]. Pretell-Mazzini et al. [8] summarized previous reports and examined the relationships between prognosis and the type of the primary tumor, gender and the number of STM; they concluded that lung cancer had a poor prognosis. Herring et al. [9] reported that there is no difference in prognosis between lung cancer and other cancers. Although the report by Pretell-Mazzini et al. [8] considered a large number of cases, they limited their examination of prognosis to 5 types of tumor, namely those of the lung, kidney, prostate, thyroid, and skin. As prostate and thyroid cancers generally provide long-term survival, lung cancer may be expected to have a poor prognosis in comparison. Herring et al. [9] included cancers with poor prognoses such as bladder and kidney cancers, gastroesophageal cancers, and melanoma. Our study did not include prostate, thyroid, or breast cancers, which offer relatively longer survival than lung can- cer; there was also no significant difference in prognosis among those who developed STM, based on the type of primary tumor. The primary tumor directly affects prognosis; large-scale studies are therefore needed to evaluate whether STM itself affects the prognosis.

STM has been widely reported to be accompanied by pain $[9,10,13,15,16]$. In the current study, $75 \%$ of the patients had pain. With respect to the association between the site of STM and pain, a previous study reported that deep-growing STM may be prone to pain [10]. In the current study, none of the patients who developed STM under the skin developed pain, whereas $92.3 \%$ of the patients who developed STM in deep layers had pain. Soft tissue sarcoma does not usually cause pain, and it is difficult to conclude whether the pain is primarily caused by tumor pressure. STM may induce pain due to other causes such as inflammation. However, the mechanism of pain in STM is completely unknown. The analysis of factors affecting prognosis demonstrated poor outcomes in those who developed STM without pain, and in those with subcutaneous STM. However, the reason for this remains unclear, and further studies are needed.

Previous studies have used various diagnostic modalities for STM. With respect to imaging, STM has been reported to present strong enhancement around the tumor on magnetic resonance imaging, and a homogenously enhancing tumor was the most common presentation in enhanced computed tomography $[11,14]$. Combining these imaging findings with pain symptoms makes it easier to distinguish primary tumors from soft tissue sarcoma. However, symptoms and imaging findings are inadequate for definitive diagnosis, and thus methods for examining primary tumors are also essential. These include histological evaluation using various immunostaining tests, including those for cytokeratin, carcinoembryonic antigen, and epithelial membrane antigen; pathological evaluation after tissue collection is also necessary $[11,12]$.

A limitation of this study was the small number of STM patients. The analysis was limited due to the small number of patients and various factors, such as types of primary tumor and treatment; this may have led to some bias. Studies with large sample sizes are needed for comprehensive multivariate analysis of the prognostic factors of STM.

\section{Conclusion}

The present study demonstrated that a history of no past treatment for the primary tumor and STM without pain were risk factors for poor prognosis in STM. In ad- 
dition, STM in the layers, such as the intramuscular or intermuscular layers, is prone to pain. More detailed studies on the diagnosis and treatment of STM are necessary in the future.

\section{Statement of Ethics}

Our study was in accordance with the ethical standards of the Helsinki Declaration of 1975, as revised in 1983. This study was approved by the Institutional Review Board for Clinical Research at Akita University, and informed consent was obtained from all patients.

\section{References}

1 Bray F, Ferlay J, Soerjomataram I, Siegel RL, Torre LA, Jemal A. Global cancer statistics 2018: GLOBOCAN estimates of incidence and mortality worldwide for 36 cancers in 185 countries. CA Cancer J Clin. 2018 Nov;68(6): 394-424.

2 Center for Cancer Control and Information Services, National Cancer Center. Projected cancer incidence in 2018. Tokyo, Japan: National Cancer Center; 2018.

3 Sridhar KS, Rao RK, Kunhardt B. Skeletal muscle metastases from lung cancer. Cancer. 1987 Apr;59(8):1530-4.

4 Chandler RW, Shulman I, Moore TM. Renal cell carcinoma presenting as a skeletal muscle mass: a case report. Clin Orthop Relat Res. 1979 Nov-Dec;(145):227-9.

5 Alexiou G, Papadopoulou-Alexiou M, Karakousis CP. Renal cell carcinoma presenting as skeletal muscle mass. J Surg Oncol. 1984 Sep; 27(1):23-5.

6 Alburquerque TL, Ortin A, Cacho J. Metastasis in deep calf muscles as first manifestation of bronchus adenocarcinoma. Am J Med. 1987 Sep;83(3):606-7.

\section{Disclosure Statement}

The authors have no conflicts of interest to declare.

\section{Author Contributions}

All authors were involved in the planning and revision of this study. H.T., H.N., K.O., M.E., Y.M., E.M., and J.S. collected the clinical data. H.T. analyzed the raw data and prepared the manuscript. N.M., K.O., T.Y., and Y.S. reviewed the manuscript.
7 Munk PL, Gock S, Gee R, Connell DG, Quenville NF. Case report 708: metastasis of renal cell carcinoma to skeletal muscle (right trapezius). Skeletal Radiol. 1992;21(1):56-9.

8 Pretell-Mazzini J, de Neyra JZ, Luengo-Alonso G, Shemesh S. Skeletal muscle metastasis from the most common carcinomas orthopedic surgeons deal with. A systematic review of the literature. Arch Orthop Trauma Surg. 2017 Nov; 137(11):1477-89.

9 Herring CL Jr, Harrelson JM, Scully SP. Metastatic carcinoma to skeletal muscle. A report of 15 patients. Clin Orthop Relat Res. 1998 Oct;355:272-81.

10 Damron TA, Heiner J. Distant soft tissue metastases: a series of 30 new patients and 91 cases from the literature. Ann Surg Oncol. 2000 Aug;7(7):526-34

11 Yoshimura Y, Isobe K, Koike T, Arai H, Aoki K, Kato H. Metastatic carcinoma to subcutaneous tissue and skeletal muscle: clinicopathological features in 11 cases. Jpn J Clin Oncol. 2011 Mar;41(3):358-64.

12 Plaza JA, Perez-Montiel D, Mayerson J, Morrison C, Suster S. Metastases to soft tissue: a review of 118 cases over a 30 -year period. Cancer. 2008 Jan;112(1):193-203.
13 Tuoheti Y, Okada K, Osanai T, Nishida J, Ehara S, Hashimoto M, et al. Skeletal muscle metastases of carcinoma: a clinicopathological study of 12 cases. Jpn J Clin Oncol. 2004 Apr; 34(4):210-4.

14 Ong N, George M, Dutta R, Ng CH. CT imaging features of skeletal muscle metastasis. Clin Radiol. 2019 May;74(5):374-7.

15 Haygood TM, Wong J, Lin JC, Li S, Matamoros A, Costelloe CM, et al. Skeletal muscle metastases: a three-part study of a not-so-rare entity. Skeletal Radiol. 2012 Aug;41(8):899909.

16 Herbowski L. Skeletal muscle metastases from papillary and follicular thyroid carcinomas: an extensive review of the literature. Oncol Lett. 2018 May;15(5):7083-9.

17 Haygood TM, Sayyouh M, Wong J, Lin JC, Matamoros A, Sandler C, et al. Skeletal Muscle Metastasis from Renal Cell Carcinoma: 21 cases and review of the literature. Sultan Qaboos Univ Med J. 2015 Aug;15(3):e327-37.

18 Glockner JF, White LM, Sundaram M, McDonald DJ. Unsuspected metastases presenting as solitary soft tissue lesions: a fourteenyear review. Skeletal Radiol. 2000 May;29(5): $270-4$. 